\title{
Bridging Jammed Grains and Glassy Atoms
}

\section{A new way to use the hard sphere model lets researchers connect the jammed states of grains and the glass transition of molecules.}

\section{by C. Patrick Royall ${ }^{1}$}

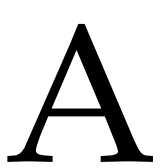
morphous solids take many forms, from glasses comprised of atoms or molecules to granular materials, such as piles of sand. At first, it is hard to imagine any similarity between these wildly different classes of materials. Atoms and molecules exhibit significant thermal motion and therefore transition between liquid, gas, and solid states of matter according to the laws of statistical mechanics. Sand grains, however, are so large that any motion due to their thermal energy is negligible, and such granular materials don't experience these phase transitions. Despite this fundamental difference, the disordered arrangements of atoms or molecules in glasses bear a striking resemblance to the rigid, jammed states that grains take on when they are compressed to sufficient density [1]. Francesco Arceri and Eric Corwin of the University of Ore-

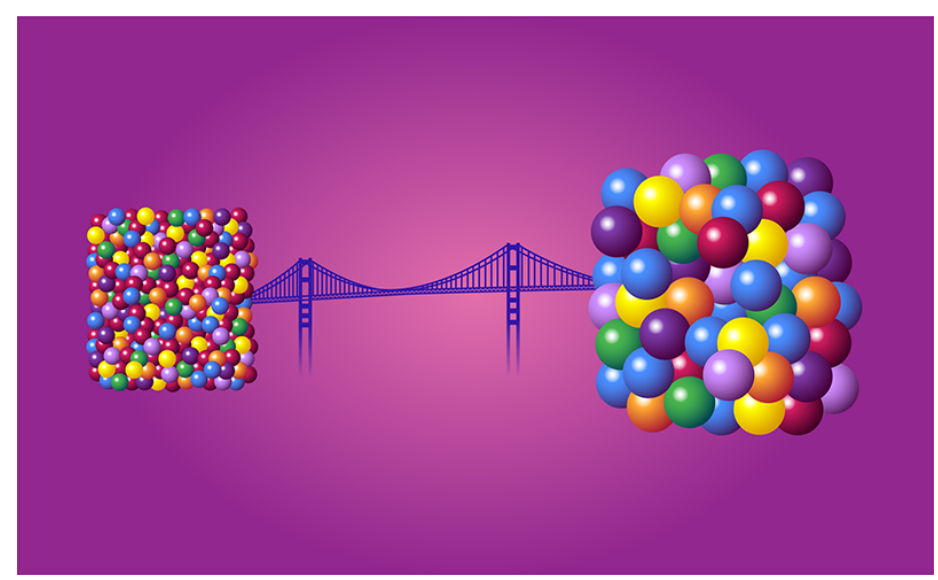

Figure 1: By realizing a way to model jammed states with simulations of hard spheres, the new work from Arceri and Corwin [2] allows direct comparisons between the behavior of assemblies of atoms or molecules (left) in glass and of jammed states made up of large grains (right), like sand. (APS/Carin Cain)

\footnotetext{
${ }^{1}$ University of Bristol, Bristol, United Kingdom
}

gon have now managed to find a new connection between the two classes of materials by using the famous hard sphere model in an unusual context so that it can simultaneously represent grains and atoms [2]. This connection opens up new comparison possibilities that weren't available before and allowed them to probe the unusual density of states of amorphous solids, which is profoundly different from that in crystalline solids.

When cooled or compressed fast enough that they fail to form ordered crystals, atoms and molecules instead form amorphous solids, or glasses-the glass transition. Such amorphous solids resemble jammed states that form when grains are packed and compressed to a certain density. This similarity was exploited by John Desmond Bernal, who used ball bearings - a granular material-in his pioneering studies that revealed so much of the structure of liquids [3]. Despite the insight that granular materials could in some ways stand in for liquids, such jammed states are fundamentally different from thermal glasses. Jamming is a "zero-temperature" phenomenon that happens to particles with negligible thermal motion, while the glass transition, where (supercooled) liquids fall out of equilibrium and become amorphous solids, occurs at finite temperature.

Given the profoundly different nature of glasses and jammed states, it is remarkable that Arceri and Corwin have managed to unite the two [2]. In their simulations, they use hard spheres as epitomized by Bernal. Beloved by theorists due to their simplicity, hard spheres models have long been a mainstay of studies of liquids and glasses. But until now, there was an apparently insurmountable problem with using hard spheres to model a jammed system.

This problem has to do with the way the two transitions are "approached" in simulations or in the lab. In a granular system, the particles will only stay together if they are packed tightly enough to jam-think of the way a pile of sand collapses unless you compress it. The jamming transition therefore has to be approached "from above," at packings higher than the jamming transition, which occurs at a packing fraction around 0.64 for similarly sized spheres. And since hard spheres cannot be squeezed together above the jamming point, soft spheres are used instead.

On the other hand, to make a glass from hard spheres, 


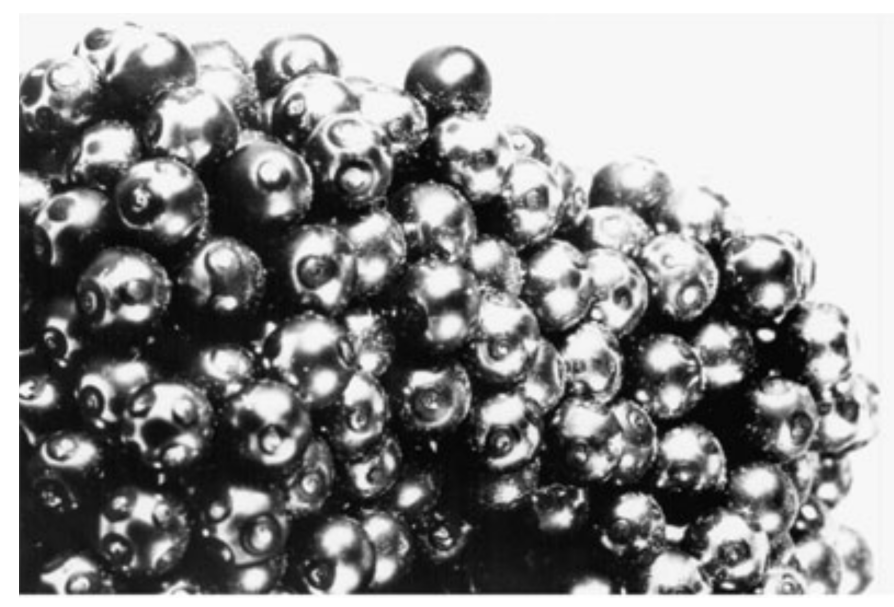

Figure 2: Bernal investigated coordination the coordination between particles in liquids by pouring paint into assemblies of ball bearings and then breaking them apart. (J. L. Finney [3])

one approaches the glass transition "from below," steadily increasing the packing fraction until the transition occurs. In addition, in hard spheres, the glass transition occurs at a lower packing fraction than jamming does [4]. This difference arises because the thermal motion in the glass makes the material become solid before the spheres actually touch one another upon compression.

In order to describe jamming with hard spheres, one needs a way to approach jamming "from below." But how could one avoid the assembly of spheres losing its rigidity and falling apart at packings less than the jamming point? Central to the problem is the "all or nothing" hard sphere interaction. As hard spheres bounce around, the interaction is zero when the particles don't touch and infinity otherwise. That is to say, the force between two hard spheres is discontinuous at the point of contact.

Crucially, theorists found a solution to this problem some years before [5]. They noted that the force between two hard spheres in a dense packing depends on the number of times the particles bounce off one another per unit time. If each particle always bounces off the same neighbors, this force should follow $1 / h$, where $h$ is the separation between the particles. Thus it is possible to find a way around the discontinuous all-or-nothing hard sphere interaction. By exploiting this loophole, Arceri and Corwin were able to effectively cool hard spheres in their simulations to almost zero temperature and treat them as a granular material, with effective interactions when the particles didn't touch one another. The system was mechanically stable at packing fractions less than jamming - so like the glass transition, it was possible to approach jamming from below.
By producing jammed packings of hard spheres, Arceri and Corwin were able to probe one of the great mysteries of amorphous solids - the density of vibrational states. The density of states of crystalline solids has been understood since the work of Debye in the early twentieth century to follow the square of the vibrational frequency. Alas, amorphous solids aren't as straightforward as the Debye model would suggest-thermal glasses exhibit a socalled boson peak of more states than expected in a certain frequency range. Jammed states similarly exhibit anomalous non-Debye behavior in their density of states. Arceri and Corwin studied the density of states of their belowjamming hard spheres and found, remarkably, that they have the same anomalous vibrational modes as soft spheres above jamming, confirming earlier theoretical predictions [6]. Moreover, the nature of these modes is the same for the hard sphere jammed packings generated by the Oregon team and for thermal glasses. Specifically, both cases exhibit vibrational modes that are spatially extended, but finite-or quasilocalized-when approached from below. This finding brings a new and unexpected link between the mechanical properties of assemblies of grains and atoms.

Being able to model jamming with hard spheres-and to approach it from below, like the glass transition-is a big step forward. It enables comparisons between jamming and the glass transition that weren't possible before when jamming was approached from above, with soft spheres. We can now return to the question Bernal raised-do the packings of granular (athermal) hard spheres correspond to those of atoms in liquids approaching the glass transition?

This research is published in Physical Review Letters.

\section{REFERENCES}

[1] A. J. Liu and S. R. Nagel, "The jamming transition and the marginally jammed solid," Annu. Rev. Condens. Matter Phys. 1, 347 (2010).

[2] F. Arceri and E. I. Corwin, "Vibrational properties of hard and soft spheres are unified at jamming," Phys. Rev. Lett. 124, 238002 (2020).

[3] J. L. Finney, "Bernal's road to random packing and the structure of liquids," Philos. Mag. 93, 3940 (2013).

[4] C. P. Royall and W. Kob, "Locally favoured structures and dynamic length scales in a simple glass-former," J. Stat. Mech. Theory Exp. 2017, 024001 (2017).

[5] C. Brito and M. Wyart, "On the rigidity of a hard-sphere glass near random close packing," Europhys. Lett. 76, 149 (2006).

[6] E. DeGiuli et al., "Theory of the jamming transition at finite temperature," J. Chem. Phys. 142, 164503 (2015).

10.1103/Physics.13.94 